\title{
Charged Exciton Dissociation Energy in (Cd,Mn)Te Quantum Wells with Variable Disorder and Carrier Density
}

\author{
A. ŁOPION $\oplus,{ }^{1,2}$ A. BOGUCKI,${ }^{1}$ K.E. POŁCZYŃSKA,${ }^{1}$ W. PACUSKI,${ }^{1}$ \\ A. GOLNIK, ${ }^{1}$ T. KAZIMIERCZUK, ${ }^{1}$ and P. KOSSACKI ${ }^{1}$ \\ 1.-Faculty of Physics, Institute of Experimental Physics, University of Warsaw, ul. Pasteura 5, \\ 02-093 Warsaw, Poland. 2.—e-mail: Aleksandra.Lopion@fuw.edu.pl
}

\begin{abstract}
We report on the magneto-photoluminescence of $(\mathrm{Cd}, \mathrm{Mn}) \mathrm{Te} /(\mathrm{Cd}, \mathrm{Mg}) \mathrm{Te}$ quantum wells excited by photons with varied energy. We observe that laser illumination modifies the carrier density and Coulomb disorder in the quantum wells. Three different regimes are analyzed, corresponding to low hole density with low disorder, low hole density with significant disorder, and high hole density. By using the diluted magnetic semiconductor as a quantum well material, we can induce the spin singlet-triplet transition of charged excitons in a magnetic field. This transition is then used as a tool to determine the charged exciton dissociation energy. With this approach, we find the same value for the dissociation energy in all the regimes of hole density and disorder. Our result is compared with the dissociation energy obtained from the PL splitting between the $X$ and $X^{+}$lines at zero field, which exhibits significantly greater variation.
\end{abstract}

Key words: Quantum well, charged exciton, trion, magnetospectroscopy, $2 \mathrm{D}$ system

\section{INTRODUCTION}

Interest in charged exciton complexes in twodimensional (2D) systems has been recently renewed due to the emergence of semiconducting transition-metal dichalcogenides (s-TMDs), for which robust excitonic resonances dominate the optical spectra. ${ }^{1-3}$ Due to the exposed surface, sTMDs are subject to significant disorder, but the strong $2 \mathrm{D}$ confinement makes it possible to observe clear narrow charged exciton complexes even for quite disordered materials. Similarly, charged exciton complexes can be observed for a wide range of carrier concentration. Both parameters, i.e., the disorder and the carrier concentration, can be easily controlled by doping or illumination of II-VI semiconductor quantum wells (QWs) ${ }^{4-8}$ For this reason, such systems provide a convenient playground to study strong excitonic effects. Here, we study the

(Received December 2, 2019; accepted April 29, 2020;

published online May 23, 2020) charged exciton dissociation energy $E_{\text {diss }}$ for various carrier concentrations and different disorders.

In our work, we exploit two particular features of II-VI QWs. The first is the unique opportunity to tune the spin polarization by applying a weak magnetic field, which is facilitated by the introduction of magnetic ions into the well material. The second is the tunability of the sample parameters by varying the optical excitation.

Generally, $(\mathrm{Cd}, \mathrm{Mn}) \mathrm{Te} /(\mathrm{Cd}, \mathrm{Mg}) \mathrm{Te} \mathrm{QWs}$ are naturally $p$-type even without intentional doping. The hole gas originates from the background doping of the $(\mathrm{Cd}, \mathrm{Mg}) \mathrm{Te}$ barrier material and/or from surface states. ${ }^{5,6}$ The former effect has been shown to be sufficiently efficient to deliver up to $2 \times 10^{12} \mathrm{~cm}^{-2}$ holes. On top of that, due to the spatial separation of the doping centers from the $\mathrm{QW}$, the carrier density might be easily tuned over several orders of magnitude by above-barrier illumination. ${ }^{4,7}$ The variable illumination of $(\mathrm{Cd}, \mathrm{Mn}) \mathrm{Te} /(\mathrm{Cd}, \mathrm{Mg}) \mathrm{Te} \mathrm{QWs}$ has also another effect of screening the electrostatic fluctuations caused by photogenerated carriers, 
thus allowing us to effectively vary the Coulomb disorder.

As established in earlier work, ${ }^{7}$ the charged excitons in $p$-doped $(\mathrm{Cd}, \mathrm{Mn}) \mathrm{Te} \mathrm{QWs}$ exhibit the singlet-triplet transition in magnetic fields of a few teslas. It was found that the energy of this transition (which is related to the charged exciton dissociation energy) is independent of the carrier density, which contradicts the expectation that the carrierdependent screening will significantly affect the dissociation energy. Additionally, the energy splitting between the $X$ and $X^{+}$lines observed in both absorption and photoluminescence, which has often been interpreted as a trion dissociation energy, is known to vary with carrier density. ${ }^{4,6,10}$ This surprising discrepancy between these two ways of determining the charged exciton dissociation energy emphasizes the importance of detailed analysis of the initial and final states. Previous works targeted the properties of delocalized charged excitons and were thus performed on samples characterized by relatively high hole densities in a wide range from $0.5 \times 10^{11} \mathrm{~cm}^{-2}$ up to $5 \times 10^{11} \mathrm{~cm}^{-2}$ with low disorder.

In this work, we apply a similar approach to explore different disorder regimes. In each studied case, the hole density is quite low, estimated to be below $10^{11} \mathrm{~cm}^{-2}$, based on the relative intensity of the $X$ and $X^{+}$photoluminescence lines. ${ }^{4,6,7}$ Crucially, the presented samples are characterized by relatively high disorder, as evidenced by the presence of localized excitonic states and broadening of the photoluminescence (PL) peak linewidths. We observe that inducing significant disorder in the samples, which results in an increase of the $X / X^{+}$spectral splitting, does not change the charged exciton dissociation energy as calculated by analysis of the singlet-triplet transition.

\section{EXPERIMENTAL PROCEDURES}

Samples containing single $(\mathrm{Cd}, \mathrm{Mn}) /(\mathrm{Cd}, \mathrm{Mg}) \mathrm{Te}$ QWs were prepared by molecular beam epitaxy (MBE). They were grown on GaAs substrate with a thick (5500 nm to $6000 \mathrm{~nm}$ ) CdTe buffer layer. $(\mathrm{Cd}, \mathrm{Mn}) \mathrm{Te} \mathrm{QWs}$ with thickness of $10 \mathrm{~nm}$ were grown between symmetrical $50-\mathrm{nm}(\mathrm{Cd}, \mathrm{Mg}) \mathrm{Te}$ barrier layers with $\mathrm{Mg}$ content of $18 \%, 22 \%$, and $20 \%$ for samples UW0676, UW1055, and UW1467, respectively. The $\mathrm{Mg}$ content in the barrier layer was determined by reflectance measurements from the bandgap energy of the barrier, yielding values of $1939 \mathrm{meV} \quad(639.2 \mathrm{~nm}), 2013 \mathrm{meV}$ $(615.75 \mathrm{~nm})$, and $1976 \mathrm{meV}(627.3 \mathrm{~nm})$, respectively. The $\mathrm{Mn}^{2+}$ content in the $\mathrm{QW}$ material of close to $0.3 \%$ was chosen to ensure sufficiently large Zeeman splitting while preserving narrow PL lines. The value was determined by fitting the modified Brillouin function ${ }^{11-13}$ to the exciton Zeeman shift obtained from reflectance measurements in a magnetic field.
The relatively small thickness of the barrier material was chosen to ensure that most of the acceptor centers would be ionized by transferring holes to either the QW or the CdTe buffer layer. Therefore, the Coulomb fluctuations in the QW are significant for a limited carrier density. Additionally, in such samples, the carrier distribution can be easily modified by choosing different illumination conditions. We discuss here three samples with similar designs but showing observably different optical properties. One of the samples (UW1055) was additionally capped with a $100-\mathrm{nm}$ CdTe layer to prevent the formation of surface states acting as acceptors.

We performed the measurements at pumped helium temperature $\approx 1.8 \mathrm{~K}$ in a magnetooptical cryostat with magnetic fields up to $3 \mathrm{~T}$. Photoluminescence was excited using different continuouswave (CW) diode lasers at selected wavelengths in the range from $457 \mathrm{~nm}$ to $647 \mathrm{~nm}$ and a tunable rhodamine dye laser. The excitation power for all the measurements was set at $5 \mu \mathrm{W}$ to avoid any thermal effects. The size of the laser spot was below $150 \mu \mathrm{m}$ in diameter. The spectra were resolved using a grating monochromator equipped with a charge-coupled device (CCD) camera.

\section{DETERMINATION OF CHARGED EXCITON DISSOCIATION ENERGY}

An important parameter characterizing the excitonic system is the charged exciton dissociation energy $E_{\text {diss }}$, i.e., the energy required to unbind the additional hole from the charged exciton complex. In this work, we extract the value of $E_{\text {diss }}$ from lowtemperature PL measurements in a magnetic field, similarly to Ref. 7.

The typical spectrum contains two main lines corresponding to the neutral exciton $(X)$ and the charged exciton complex (identified further as a positively charged exciton $X^{+}$), as seen in Fig. 1a. Additionally, a tail of localized states is visible for samples with higher disorder in the energy range below the charged exciton line. ${ }^{14}$

At first glance, it may seem that a convenient measure of $E_{d i s s}$ would be the distance between the $X$ and $X^{+}$lines in the PL spectrum. However, this reasoning does not take into account that the final states in the recombination processes for $X$ and $X^{+}$ might be different. In particular, the carrier gas left by the recombination of $X^{+}$might be excited, which would lead to an apparent increase of the splitting between $X$ and $X^{+}$. Another possibility resulting in an increase of this splitting is the localization of the $X^{+}$complex. The extreme case of such localization is formation of the $D^{0} X$ or $A^{0} X$ complexes, ${ }^{15}$ which have over two times larger binding energy than free $X^{+}$complexes. On the other hand, in the simple picture, the $X^{+}$transition energy should not be sensitive to the Coulomb disorder, which potentially 
(a)
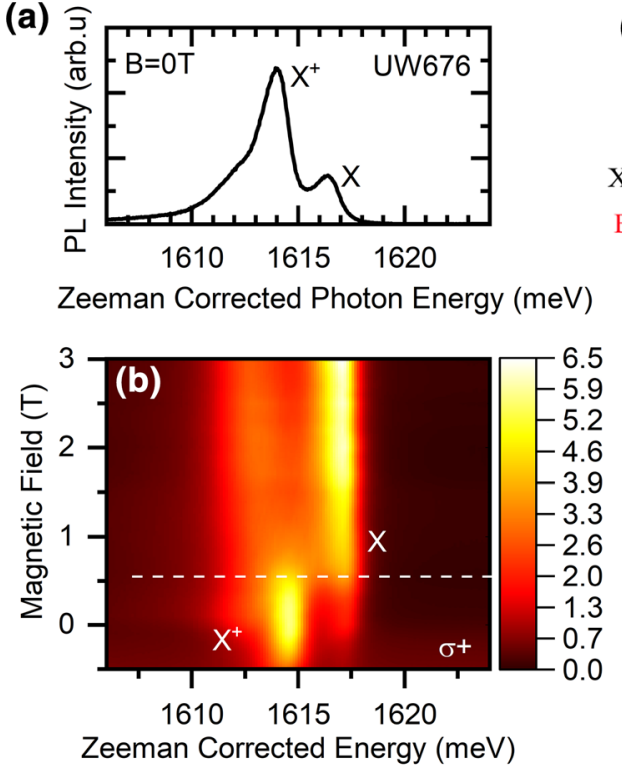

(c)



(d)

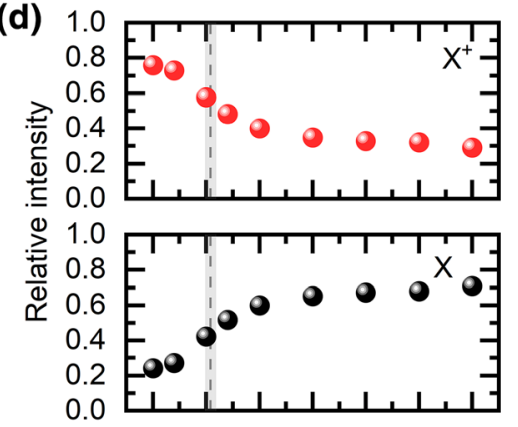

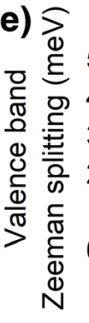

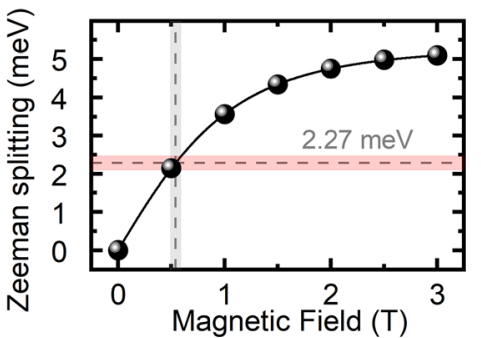

Fig. 1. Results for sample UW0676: (a) Low-temperature PL spectrum. (b) PL spectrum in magnetic field corrected by Zeeman shift similar to (e), with spin singlet-triplet transition present at magnetic field of approximately $0.5 \mathrm{~T}$. (c) Diagram of optical transitions in the positively charged system containing heavy hole $(\mathrm{hh})$, positively charged exciton $\left(X^{+}\right)$, and neutral exciton $(X)$; single arrows represent electrons, and double arrows indicate holes; red arrows represent charged exciton dissociation energy equal to heavy hole splitting in magnetic field $B_{0}$; blue arrows represent Zeeman splitting; dashed line marks the level crossing connected to the spin singlet-triplet transition. (d) Peak intensity of $X$ and $X^{+}$as part of total peak intensity of both excitonic lines measured for $\sigma^{+}$polarization in the magnetic field under excitation by 561 -nm laser. (e) Valence-band Zeeman splitting obtained by multiplying the measured neutral exciton Zeeman splitting by $N_{0} \beta / N_{0}(\beta-\alpha)=0.8$; shaded areas in panels (d) and (e) represent the error estimation of determined values: grey for $B_{0}$ and faded red for $E_{\text {diss }}$ (Color figure online).

undermines the link between $E_{\text {diss }}$ and the spectral distance between $X$ and $X^{+}$. To avoid this difficulty, we employed another approach to determine $E_{\text {diss }}$ based on measurements in a magnetic field.

In a magnetic field, both the neutral and charged exciton lines exhibit the giant Zeeman shift. Figure $1 \mathrm{~b}$ shows a map of the PL spectrum of one of the samples (UW0676) as a function of the magnetic field. The measurement was carried out under excitation at wavelength of $\lambda=561 \mathrm{~nm}$. Energy in the figure is corrected by the Zeeman shift to highlight the evolution of the spectra. At a magnetic field of approximately $0.55 \mathrm{~T}$, the intensity of the charged exciton line rapidly decreases with a simultaneous increase of the intensity of the neutral exciton line. This transition is caused by the crossing of energy levels in the magnetic field (Fig. 1c). At this point, the $X^{+}$state (spin singlet) is crossed by the neutral exciton-hole triplet state. ${ }^{15-18}$

The magnetic field value at the singlet-triplet crossing can provide additional information about the studied system. For instance, it allows us to identify the majority carriers in the sample. This stems from the fact that the exchange parameters for electrons and holes are $N_{0} \alpha=0.22 \mathrm{eV}$ and $N_{0} \beta=-0.88 \mathrm{eV}$, respectively, where $N_{0}$ denotes the number of unit cells per unit volume and $\alpha=$ $\langle s|J| s\rangle$ and $\beta=\langle X|J| X\rangle$ are the exchange integrals for the conduction and valence band. ${ }^{11,12}$ In our case, the spin singlet-triplet transition is observed at a relatively low magnetic field (below $1 \mathrm{~T}$ ), which unequivocally identifies the charged exciton as the positively charged $X^{+}$and establishes the majority of carriers as holes.

Deeper analysis allows us to additionally relate the observed singlet-triplet crossing to the value of $E_{\text {diss }}$. As presented in Fig. 1c, the Zeeman shift of energy levels participating in this process is given by $1 / 2 x N_{0} \alpha\left\langle S_{M n}^{z}\right\rangle$ for the charged exciton and $-1 / 2 x N_{0}(2 \beta-\alpha)\left\langle S_{M n}^{z}\right\rangle$ for the triplet state of the neutral exciton accompanied by an unbound hole, ${ }^{11,12}$ where $x\left\langle S_{M n}^{z}\right\rangle$ denotes the mean value of the spin per cation site. The field of the crossing $B_{0}$ corresponds to the situation when the two levels are degenerate. Taking into account the zero-field splitting between $X$ and $X^{+}$states (i.e., $E_{\text {diss }}$ ):

$$
E_{d i s s}-1 / 2 x N_{0}(2 \beta-\alpha)\left\langle S_{M n}^{z}\right\rangle B_{0}=1 / 2 x N_{0} \alpha\left\langle S_{M n}^{z}\right\rangle B_{0},
$$

which leads to

$$
E_{\text {diss }}=N_{0} \beta\left\langle S_{M n}^{z}\right\rangle B_{0}
$$

Coincidentally, the right-hand side of Eq. 2 describes the Zeeman splitting of the valence band at a magnetic field of $B_{0}{ }^{7}$ In turn, its value can be calculated from the Zeeman splitting of the neutral exciton $\Delta E_{X}$, which is directly accessible in the experiment: 


$$
\begin{aligned}
E_{\text {diss }} & =\frac{N_{0} \beta}{N_{0}(\beta-\alpha)} N_{0}(\beta-\alpha)\left\langle S_{M n}^{z}\right\rangle B_{0} \\
& =\frac{N_{0} \beta}{N_{0}(\beta-\alpha)} \Delta E_{X}=\frac{4}{5} \Delta E_{X} .
\end{aligned}
$$

The obtained relation gives us a practical method to determine the value of $E_{\text {diss }}$ as $80 \%$ of the neutral exciton splitting at the point of the singlet-triplet transition. This approach has a clear advantage over the previously discussed distance between the $X$ and $X^{+}$lines: here we inherently compare the energies of neutral and charged excitons at the same position of the possibly disordered energy landscape.

\section{RESULTS AND DISCUSSION}

Figure 1d presents the integrated intensity of the $X^{+}$and $X$ lines divided by the total QW emission. The singlet-triplet transition field $B_{0}$ is tentatively defined as the field at which the relative emission intensity is the average of the levels of its high and low plateaux. From the determination of $B_{0}$, the $X^{+}$ dissociation energy can be derived, as presented in Eq. 1 (Fig. 1e). The uncertainties on the derived values are based on the uncertainty of determining the low and high plateau levels.

We performed PL measurements with excitation wavelengths varying from $457 \mathrm{~nm}$ to $647 \mathrm{~nm}$ and the same excitation laser intensity for the three different samples presented in "Experimental Procedures" section. Consistent with previous studies, we observed that changes in the excitation wavelength led to a gradual change in the relative intensities of the $X$ and $X^{+}$lines in the PL spectrum, which is interpreted as a signature of variations of the carrier density. In the high carrier density regime, the PL spectrum is dominated by the charged exciton line with the absence of neutral exciton. With increasing excitation wavelength, the system enters the low hole density regime and the neutral exciton becomes visible. Note that the singlet-triplet transition was clearly visible for all excitation conditions. Even if the neutral exciton is absent from the PL spectra at $B=0 \mathrm{~T}$, upon reaching the threshold value of magnetic field, the $X^{+}$line disappears and another line emerges close to the position of the neutral exciton. The identification of the singlet-triplet transition allowed us to apply the approach from "Determination of Charged Exciton Dissociation Energy" section to determine $E_{\text {diss }}$ for each of the three samples, which we discuss below in detail.

Figure 2 presents PL spectra of sample UW0676 measured in the absence of a magnetic field. For short excitation wavelength, the charged exciton line dominates the spectrum and the neutral exciton line is not visible, which indicates high carrier density in the QW. With increasing excitation wavelength, we observe a decrease of the carrier

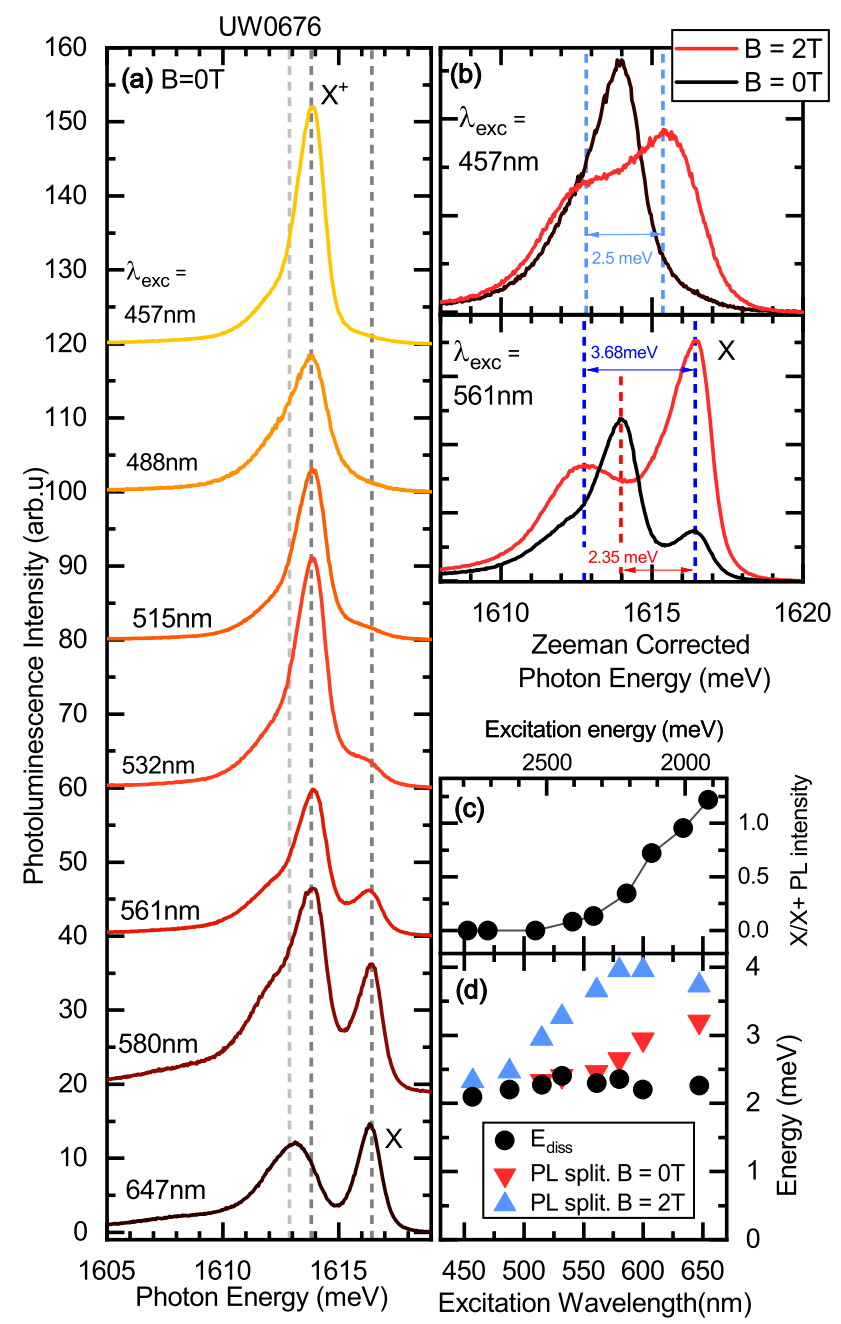

Fig. 2. Results for sample UW0676: (a) PL spectra excited at different wavelengths. On excitation at wavelength of $457 \mathrm{~nm}$, charged exciton $X^{+}$is visible, and its PL decreases with increase of the excitation wavelength. For longer excitation wavelengths, localized state is revealed. (b) Comparison of PL spectra in magnetic field of $0 \mathrm{~T}$ and $2 \mathrm{~T}$ (corrected by Zeeman splitting) for excitation wavelengths of $457 \mathrm{~nm}$ and $561 \mathrm{~nm}$. (c) Relative intensity of neutral exciton and charged exciton for full range of excitation wavelengths. (d) Splitting of PL lines and $E_{\text {diss }}$ observed for different excitation wavelengths.

density as evidenced by the appearance of the neutral exciton line shown in Fig. 2a. The energy distance between the maxima of the $X$ and $X^{+}$ emission lines is about $2.5 \mathrm{meV}$ for short excitation wavelengths and increases for longer excitation wavelength to $3.5 \mathrm{meV}$ to $4 \mathrm{meV}$, as shown by red points in Fig. $2 d$. This change may reflect the increasing contribution of localized states in the $X^{+}$emission line, possibly linked to the presence of an additional broad PL peak at lower energy, which becomes more pronounced upon weakening of the positively charged exciton $X^{+}$PL signal with decreasing carrier density (Fig. 2a).

The magnetooptical measurements show the spin singlet-triplet transition for excitation in the whole 
studied range. In particular, the spectra obtained in a magnetic field for high hole gas density (excitation at $457 \mathrm{~nm}$ ) resemble spectra previously reported for QWs containing a degenerate spin-polarized hole gas $^{7}$ with a clearly pronounced singlet-triplet transition. The $E_{\text {diss }}$ extracted from the value of the transition field is essentially constant for all the studied excitation wavelengths, as shown by the black points in Fig. 2 d.

To analyze the case of larger disorder with low carrier density, we studied a sample with a $100-\mathrm{nm}$ CdTe cap on top of the upper barrier layer. This cap prevents the formation of acceptor surface states,, 19 while narrow barrier layers result in ionization of the background acceptors in the barrier material, which should create Coulomb potential fluctuations in the quantum well. As expected, the low hole density regime was observed in the whole range of studied excitation wavelengths, and the neutral exciton was always present in the absence of a magnetic field, as shown in Fig. 3a, b. For all the studied excitation wavelengths, the energy distance between the neutral exciton line and the center of the $X^{+}$line was relatively large, approximately $3 \mathrm{meV}$ to $4 \mathrm{meV}$. This is consistent
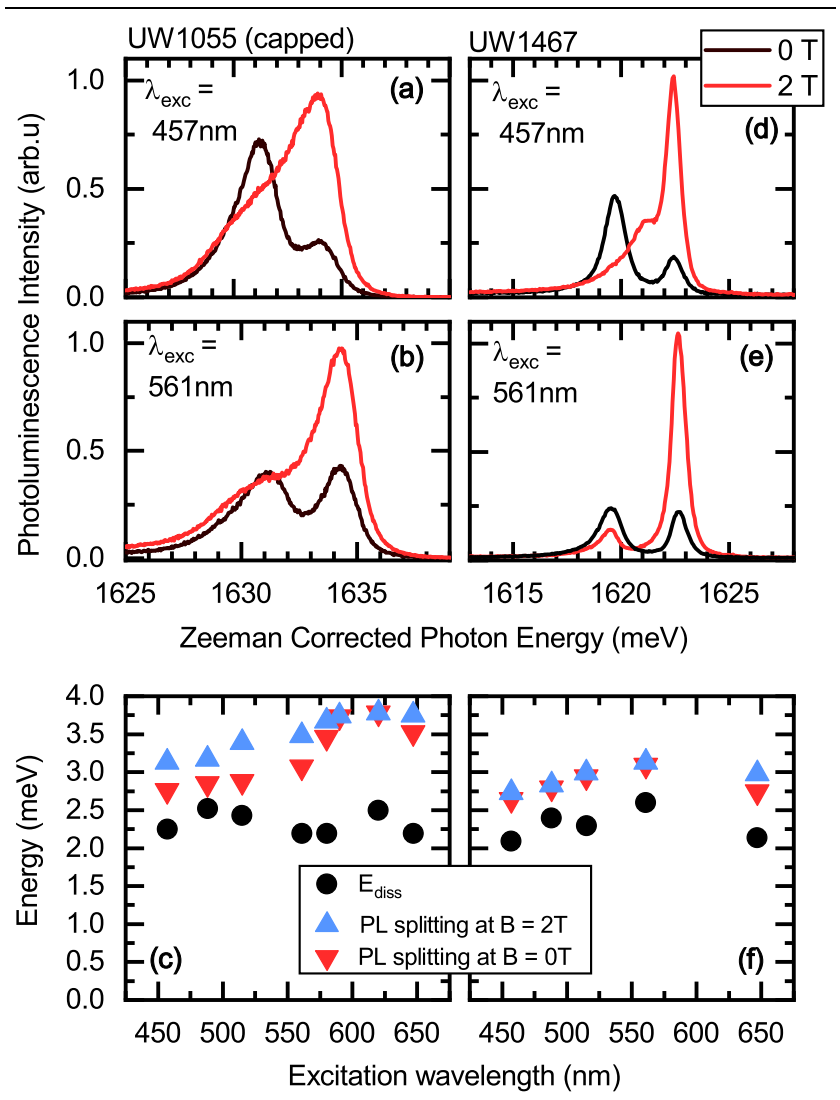

Fig. 3. PL spectra measured for capped UW1055 sample at magnetic field of $0 \mathrm{~T}$ and $2 \mathrm{~T}$ for excitation wavelength of (a) $457 \mathrm{~nm}$ and (b) $561 \mathrm{~nm}$. (c) Splitting of PL lines and $E_{\text {diss }}$ observed for different excitation wavelengths for UW1055 sample. (d-f) Corresponding data for sample UW1467. with the assumption that the contribution of the localized states in the $X^{+}$line is greater than in the case of the sample discussed above. Despite the fact that the sample is always in the low carrier density regime and the localization is deeper for UW1055, the spin singlet-triplet transition was still observable. It was used to determine a hole dissociation energy at the level of about $2.2 \mathrm{meV}$, similarly to the values obtained for sample UW0676 above. Again, as shown in Fig. $3 \mathrm{~b}$, the value of $E_{\text {diss }}$ determined using the singlet-triplet transition is essentially constant for different excitation wavelengths, while the spectral separation of the $X$ and $X^{+}$lines clearly increases for longer excitation wavelengths. This finding invalidates the simplest picture in which both the zero-field splitting and singlet-triplet transition reflect the actual $E_{\text {diss }}$ energy affected by the enhancement of the $X$ and $X^{+}$binding energies due to localization, analogously to such an effect in $D^{0} X$ and $A^{0} X$.

The third studied sample was similar to the first one, but grown in conditions that provided very low background doping (UW1467). As seen in Fig. 3c, d, the PL lines for both neutral and charged excitons were very narrow (full-width at half-maximum, FWHM $\sim 1 \mathrm{meV}$ ). Similarly, as in the case of sample UW1055, the neutral exciton was present in $0 \mathrm{~T}$ magnetic field, and we can assume that this sample corresponds to the low carrier density regime. The spin singlet-triplet transition is observed at magnetic fields similar to the previous cases, but in this particular case, the PL of the charged exciton is completely quenched, and we cannot observe any localized state at lower energy. Thus, we can assume that there is no effect of localization of charged excitons and that this sample is characterized by low disorder. Moreover, in the energy range between charged and neutral excitons, we found another state, which is revealed as the charged exciton vanishes. Overall, regarding the charged exciton dissociation energy, this sample confirms the trends observed for the previous samples; namely, the zero-field energy distance between charged and neutral excitons (in the range from $2.6 \mathrm{meV}$ to $3.2 \mathrm{meV}$ ) slightly increases with the excitation wavelength. The $E_{d i s s}$ is at a level of about $2.2 \mathrm{meV}$, as in the previous cases. The values determined for the studied excitation wavelengths are collected in Fig. 3d.

Note that the fact that all the measured samples were characterized by the same $E_{\text {diss }}$ value of about $2.2 \mathrm{eV}$ does not imply that they behave identically in a magnetic field. Depending on the particular sample and excitation wavelength, PL measurements in a magnetic field yield different effective $g$ factor values, as well as different threshold fields for the singlet-triplet splitting, whille only their combination (i.e., $E_{\text {diss }}$ ) turns out to produce a constant value, as illustrated in Fig. 4. 


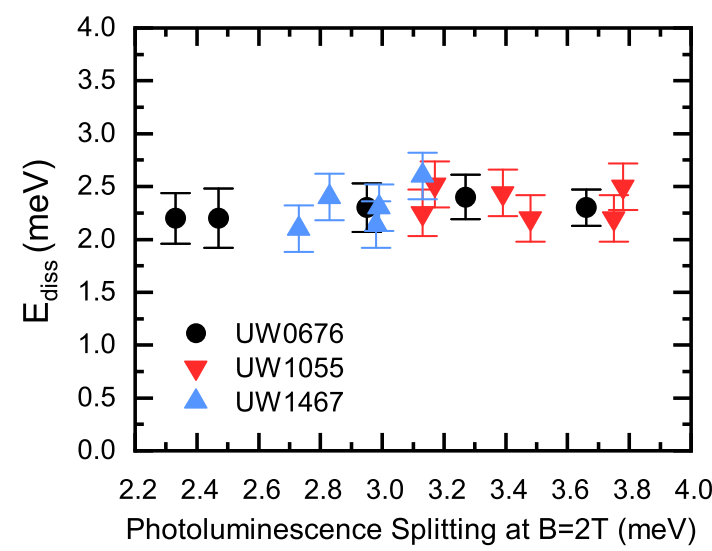

Fig. 4. Charged exciton dissociation energy as function of PL line splitting, measured at $2 \mathrm{~T}$ magnetic field.

\section{CONCLUSIONS}

We find that the positively charged exciton dissociation energy, defined as the energy necessary to unbind an additional hole by flipping its spin, is constant for a broad range of parameters such as carrier density and Coulomb fluctuations, despite the large impact of those parameters on the observed distance between the $X$ and $X^{+}$lines in PL spectra.

\section{ACKNOWLEDGMENTS}

This work was supported by the Polish National Science Centre under decisions DEC-2016/23/B/ ST3/03437. P.K., A.B., and T.K. were supported by the ATOMOPTO project (TEAM program of the Foundation for Polish Science) cofinanced by the EU within the ERDFund. The project was carried out with the use of CePT, CeZaMat, and NLTK infrastructures financed by the European Union - the European Regional Development Fund within the Operational Programme "Innovative economy" for 2007-2013.

\section{OPEN ACCESS}

This article is licensed under a Creative Commons Attribution 4.0 International License, which permits use, sharing, adaptation, distribution and reproduction in any medium or format, as long as you give appropriate credit to the original author(s) and the source, provide a link to the Creative Commons licence, and indicate if changes were made. The images or other third party material in this article are included in the article's Creative Commons licence, unless indicated otherwise in a credit line to the material. If material is not included in the article's Creative Commons licence and your intended use is not permitted by statutory regulation or exceeds the permitted use, you will need to obtain permission directly from the copyright holder. To view a copy of this licence, visit http://creativecom mons.org/licenses/by/4.0/.

\section{REFERENCES}

1. K.F. Mak, K. He, C. Lee, G.H. Lee, J. Hone, T.F. Heinz, and J. Shan, Nat. Mater. 12, 207 (2013).

2. M. Sidler, P. Back, O. Cotlet, A. Srivastava, T. Fink, M. Kroner, E. Demler, and A. Imamoglu, Nat. Phys. 13, 255 (2017).

3. M. Goryca, J. Li, A.V. Stier, T. Taniguchi, K. Watanabe, E. Courtade, S. Shree, C. Robert, B. Urbaszek, X. Marie, and S.A. Crooker, Nat. Commun. 10, 1 (2019).

4. P. Kossacki, J. Cibert, D. Ferrand, Y.M. Aubigne, A. Arnoult, A. Wasiela, S. Tatarenko, and J. Gaj, Phys. Rev. B 60, 16018 (1999)

5. W. Maślana, P. Kossacki, M. Bertolini, H. Boukari, D. Ferrand, S. Tatarenko, J. Cibert, and J. Gaj, Appl. Phys. Lett. 82,1875 (2003).

6. P. Kossacki, J. Phys. Condens. Matter 15, R471 (2003).

7. P. Kossacki, H. Boukari, M. Bertolini, D. Ferrand, J. Cibert, S. Tatarenko, J. Gaj, B. Deveaud, V. Ciulin, and M. Potemski, Phys. Rev. B 70, 195337 (2004).

8. C. Aku-Leh, F. Perez, B. Jusserand, D. Richards, W. Pacuski, P. Kossacki, M. Menant, and G. Karczewski, Phys. Rev. B 76, 155416 (2007)

9. M. Bertolini, W. Maslana, H. Boukari, B. Gilles, J. Cibert, D. Ferrand, S. Tatarenko, P. Kossacki, and J. Gaj, J. Cryst. Growth 251, 342 (2003).

10. V. Huard, R. Cox, K. Saminadayar, A. Arnoult, and S. Tatarenko, Phys. Rev. Lett. 84, 187 (2000).

11. J.A. Gaj and J. Kossut, Introduction to the Physics of Diluted Magnetic Semiconductors, vol. 144, (Springer, Berlin, 2011).

12. J. Gaj, R. Planel, and G. Fishman, Solid State Commun. 29, 435 (1979).

13. J. Gaj, W. Grieshaber, C. Bodin-Deshayes, J. Cibert, G. Feuillet, Y.M. Aubigne, and A. Wasiela, Phys. Rev. B 50, 5512 (1994).

14. V. Ciulin, P. Kossacki, S. Haacke, J.-D. Ganiere, B. Deveaud, A. Esser, M. Kutrowski, and T. Wojtowicz, Phys. Rev. $B$ 62, R16310 (2000).

15. K. Kheng, R. Cox, M.Y. Aubigne, F. Bassani, K. Saminadayar, and S. Tatarenko, Phys. Rev. Lett. 71, 1752 (1993).

16. G. Astakhov, D. Yakovlev, V. Kochereshko, W. Ossau, J. Nürnberger, W. Faschinger, and G. Landwehr, Phys. Rev. B 60, R8485 (1999).

17. G. Astakhov, D. Yakovlev, V. Kochereshko, W. Ossau, W. Faschinger, J. Puls, F. Henneberger, S. Crooker, Q. McCulloch, D. Wolverson, N.A. Gippius, and A. Waag, Phys. Rev. B 65, 165335 (2002).

18. G. Finkelstein, V. Umansky, I. Bar-Joseph, V. Ciulin, S. Haacke, J.-D. Ganiere, and B. Deveaud, Phys. Rev. B 58, 12637 (1998).

19. S. Tatarenko, M. Bertolini, W. Maslana, H. Boukari, B. Gilles, J. Cibert, D. Ferrand, P. Kossacki, and J.A. Gaj, in Solid State Crystals 2002: Crystalline Materials for Optoelectronics, vol. 5136, (International Society for Optics and Photonics, Bellingham, 2003), pp. 249-255.

Publisher's Note Springer Nature remains neutral with regard to jurisdictional claims in published maps and institutional affiliations. 